\title{
Uncertainty in optical bio-sensors due to the spectral displacement of the interference modes of the transduction signal
}

\section{Incertidumbre en bio-sensores ópticos asociada al desplazamiento espectral de los modos de interferencia de la señal de transducción}

\author{
J. de Vicente $\left(1,{ }^{*}\right)$, M. Holgado(1,2), A. Lavín(1), F. J. Sanza(2), R. Casquel(2), M. F. Laguna(2) \\ 1. Dpto. Física Aplicada, ETS Ingenieros Industriales, Universidad Politécnica de Madrid C/ José Gutiérrez Abascal, 2 - \\ 28006 Madrid, Spain \\ 2. Centro Láser, Universidad Politécnica de Madrid, Ed. Tecnológico "La Arboleda", Campus Sur UPM, Ctra. Valencia, \\ km. 7,3 - 28031 Madrid, Spain \\ (*) Email: jvo@etsii.upm.es \\ S: miembro de SEDOPTICA / SEDOPTICA member \\ Received / Recibido: 19/09/2013. Revised / Revisado: 18/12/2013. Accepted / Aceptado: 08/01/2014. \\ DOI: http://dx.doi.org/10.7149/OPA.47.1.27
}

\begin{abstract}
:
The analysis of the interference modes has an increasing application, especially in the field of optical biosensors. In this type of sensors, the displacement $\Delta v$ of the interference modes of the transduction signal is observed when a particular biological agent is placed over the biosensor. In order to measure this displacement, the position of a maximum (or a minimum) of the signal must be detected before and after placing the agent over the sensor. A parameter of great importance for this kind of sensors is the period $P_{v}$ of the signal, which is inversely proportional to the optical thickness $\mathrm{h} 0$ of the sensor in the absence of the biological agent. The increase of this period improves the sensitivity of the sensor but it worsens the detection of the maximum. In this paper, authors analyze the propagation of uncertainties in these sensors when using least squares techniques for the detection of the maxima (or minima) of the signal. Techniques described in supplement 2 of the ISOGUM Guide are used. The result of the analysis allows a metrological educated answer to the question of which is the optimal period $P_{v}$ of the signal.
\end{abstract}

Key words: Biosensors, Optical Interrogation, Metrology, Measurement, Uncertainty.

\section{RESUMEN:}

El análisis del comportamiento de los modos de interferencia tiene una aplicación cada vez más amplia, especialmente en el campo de los biosensores ópticos. En este tipo de sensores se observa el desplazamiento $\Delta v$ de los modos de interferencia de la señal de transducción al reconocer un determinado agente biológico. Para medir ese desplazamiento se debe detectar la posición de un máximo o mínimo de la señal antes y después de dicho desplazamiento. En este tipo de biosensores un parámetro de gran importancia es el periodo $P_{v}$ de la señal el cual es inversamente proporcional al espesor óptico h0 del sensor en ausencia de agente biológico. El aumento de dicho periodo mejora la sensibilidad del sensor pero parece dificultar la detección del mínimo o máximo. Por tanto, su efecto sobre la incertidumbre del resultado de la medida presenta dos efectos contrapuestos: la mejora de la sensibilidad frente a la dificultad creciente en la detección del mínimo ó máximo. En este trabajo, los autores analizan la propagación de incertidumbres en estos sensores utilizando herramientas de ajuste por MM.CC. para la detección de los mínimos o máximos de la señal y técnicas de propagación de incertidumbres descritas en el suplemento 2 de la Guía ISO-GUM. El resultado del análisis permite dar una respuesta, justificada desde el punto de vista metrológico, de en que condiciones es conveniente o no aumentar el periodo $P_{v}$ de la señal.

Palabras clave: Biosensores, Interrogación Óptica, Metrología, Medida, Incertidumbre. 


\section{REFERENCES AND LINKS / REFERENCIAS Y ENLACES}

[1]. BIPM - Bureau International des Poids et Mesures, "An introduction to the GUM and related documents", Joint Committee for Guides in Metrology, JCGM 104:2009.

http://www.bipm.org/en/publications/guides/gum.html

[2]. BIPM - Bureau International des Poids et Mesures, "Supplement 2 to the GUM - Extension to any number of output quantities", Joint Committee for Guides in Metrology, JCGM 102:2011.

[3]. BIPM - Bureau International des Poids et Mesures, "Evaluation of measurement data - Guide to the expression of uncertainty in measurement", Joint Committee for Guides in Metrology, JCGM 100:2008

[4]. M. Holgado, C. A Barrios, F. J. Ortega, F. J. Sanza, R. Casquel, M. F. Laguna, M. J. Bañuls, D. LópezRomero, R. Puchades, A. Maquieira, "Label-free biosensing by means of periodic lattices of high aspect ratio SU-8 nano-pillars”, Biosens. Bioelectron. 25, 2553-2558 (2010). DOI

[5]. F. J. Sanza, M. Holgado, F. J. Ortega, R. Casquel, D. López-Romero, M. J. Bañuls, M. F. Laguna, C. A. Barrios, R. Puchades, A. Maquieira, "Bio-photonic sensing cells over transparent substrates for anti gestrinone antibodies biosensing", Biosens. Bioelectron. 26, $4842-4847$ (2011). DOI

[6]. A. Björck, Numerical Methods for Least Squares Problems, SIAM Ed., Philadelphia (1996). DOI

[7] BIPM- Bureau International des Poids et Mesures, "International vocabulary of metrology - Basic and general concepts and associated terms", Joint Committee for Guides in Metrology, JCGM 200:2012

[8]. A. Lavín, R. Casquel, F. Sanza, M. F. Laguna, M. Holgado, "Efficient design and optimization of biophotonic sensing cells (BICELLs) for label free biosensing", Sensor. Actuat. B - Chem. 176, 753-760 (2013).

\section{Introducción}

Actualmente se considera que hay una gran necesidad de desarrollar herramientas de diagnóstico capaces de asegurar sensibilidad, rapidez, sencillez y asequibilidad para aplicaciones en sectores como la salud, la alimentación, el medioambiente o la seguridad. En el ámbito clínico se necesitan profundos avances tecnológicos capaces de ofrecer análisis rápidos, con baja incertidumbre, fiables $\mathrm{y}$ asequibles en coste $y$ que tengan como consecuencia la mejora clínica y económica a partir de un diagnóstico eficiente. En concreto, hay un interés creciente por la descentralización del diagnóstico clínico mediante plataformas de detección cercanas al usuario final, denominadas POCs (Point Of Care devices). La utilización de POCs (referidas al diagnóstico cercano al usuario final o fuera del laboratorio de análisis clínico), mediante detección in vitro (IVD) será extremadamente útil en centros de salud, clínicas o unidades hospitalarias, entornos laborales o incluso en el hogar.

Por otra parte, el desarrollo de la genómica, proteómica y otras tecnologías conocidas como "omics" (sufijo inglés utilizado para referirse, por ejemplo, a genomics, transcriptomics, proteomics, metabolomics, lipidomics) está incrementando la demanda de nuevas tecnologías con una clara orientación hacia la medicina y el diagnóstico personalizado con el objeto de hacer frente a cambios en los tratamientos en el caso de enfermedades complejas.

Para desarrollar los sistemas de biodetección, es necesario conjuntar varias disciplinas científicas que van desde la micro y nano tecnología a las técnicas eficientes de interrogación óptica, biometrología y toda la inmunoquímica asociada a la inmovilización de bio-receptores y el reconocimiento selectivo de sustancias.

Los chips desarrollados en el Centro Laser de la UPM están basados en celdas biosensoras, que se han denominado BICELLs [4,5]. Cada celda puede estar programada para detectar una determinada sustancia o elemento extraño. Cuanto menor sea el tamaño de estas celdas, mayor será la capacidad de analizar múltiples analitos de forma simultánea y menor será el coste por análisis. El sistema ofrecerá ventajas muy competitivas en comparación con las técnicas de análisis clásicas. Entre otras, cabe destacar la rapidez en el tiempo de análisis. La tecnología permitirá desarrollar biochips de bajo 
coste para análisis cercanos al usuario final y biotarjetas para monitorización más eficiente de terapias y tratamientos. Con esta tecnología se facilitará el acceso a la prevención y se acercará el diagnóstico al usuario final, de tal forma que la transferencia de esta tecnología tendrá un elevado impacto social. Por otra parte, es destacable el trabajo realizado en el diseño de plataformas de lectura de los biochips. Las plataformas de lectura de los biochips son una parte esencial ya que son las encargadas de traducir la información generada por los biochips y servir de interfaz con el usuario final.

\section{Principio de funcionamiento}

El procedimiento que utilizan estos biosensores para la detección de la presencia del agente biológico es la observación del desplazamiento $\Delta v$ en frecuencia de los modos de interferencia de la señal luminosa que incide sobre la muestra al depositarse una cierta cantidad de dicho agente sobre el biosensor (Fig. 1).

Dicha señal interferencial se genera del siguiente modo: un haz de luz blanca (Fig. 2) se hace incidir, con un ángulo $\theta$, sobre un interferómetro sencillo tipo Fabry-Perot compuesto por un substrato (medio 3) y una lámina delgada (medio 2) de espesor $z_{0} \mathrm{y}$ con diferente índice de refracción que el substrato. Sobre dicha lámina delgada se deposita el agente biológico y al hacerlo se puede considerar que el espesor $z$ de la lámina aumenta, pudiendo

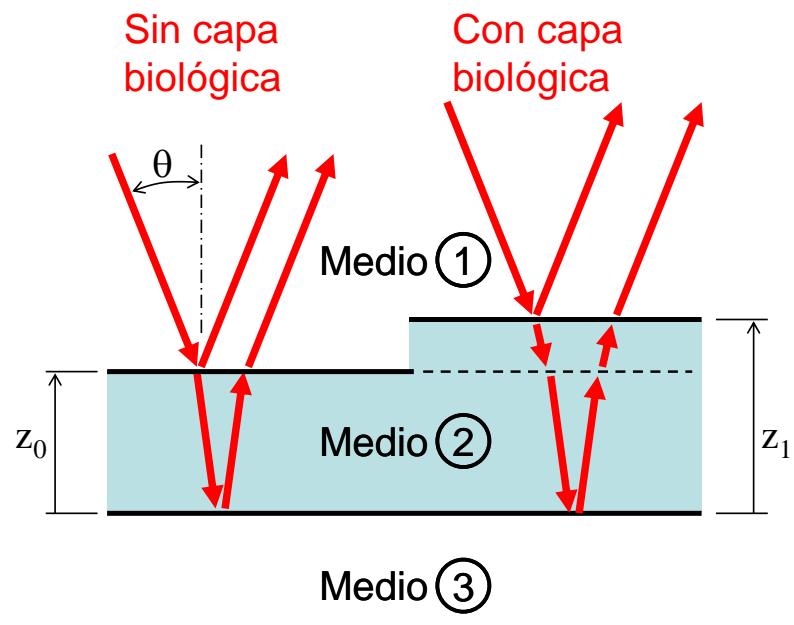

además variar el índice de refracción $n$ de este medio, y por ende se produce una variación en el espesor óptico efectivo $h$ [8]. En nuestro modelo hemos considerado que el índice de la capa biológica es similar al de la lámina delgada del interferómetro (p.e. una simple capa de óxido de silicio de índice de refracción similar al de una capa biológica y del orden de 1,4). Esta aproximación nos permite simplificar el modelo y los cálculos.

La intensidad del haz reflejado se mide a diferentes frecuencias $v$ (o longitudes de onda $\lambda=c / v$, siendo $c$ la velocidad de la luz). Hallando el cociente entre la intensidad reflejada

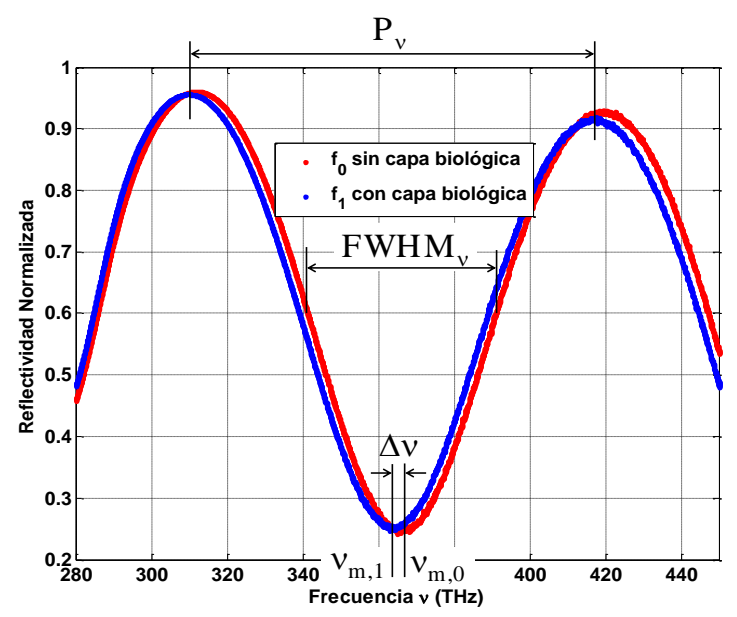

Fig. 1. Desplazamiento de la señal interferencial.

$$
\begin{aligned}
& \begin{array}{l}
\text { Espesor } \\
\text { Óptico } \\
\text { Efectivo }
\end{array} \begin{cases}\text { sin capa biológica } & \mathrm{h}_{0}=\int_{0}^{z_{0}} \mathrm{n}(\mathrm{z}) \mathrm{dz} \\
\text { con capa biológica } & \mathrm{h}_{1}=\int_{0}^{z_{1}} \mathrm{n}(\mathrm{z}) \mathrm{dz}\end{cases} \\
& \mathrm{h}_{1}=\int_{0}^{\mathrm{z}_{1}} \mathrm{n}(\mathrm{z}) \mathrm{dz}=\int_{0}^{\mathrm{z}_{0}} \mathrm{n}(\mathrm{z}) \mathrm{dz}+\overbrace{\int_{\mathrm{z}_{0}}^{z_{1}} \mathrm{n}(\mathrm{z}) \mathrm{dz}}^{\Delta \mathrm{h}}=\mathrm{h}_{0}+\Delta \mathrm{h}
\end{aligned}
$$

Fig. 2: Esquema de un biosensor no estructurado. 
y la intensidad del haz incidente se puede calcular la reflectividad $R$ de la lámina delgada. Dicha reflectividad $R$ es función tanto del espesor óptico $h$ como de la frecuencia $v$ (o de la longitud de onda $\lambda$ ):

$$
R(v, h)=R_{\text {med }}+\frac{A}{2} \cos \left(\phi_{v}\right)=,
$$

donde,

$$
\phi_{v}=2 \pi \frac{v}{c /(2 h \cos \theta)}
$$

ó

$$
R(\lambda, h)=R_{\text {med }}+\frac{A}{2} \cos \left(\phi_{\lambda}\right),
$$

donde

$$
\phi_{\lambda}=2 \pi \frac{h}{\lambda / 2} \cos \theta
$$

y donde $R_{\text {med }}$ es el valor medio de la reflectividad y $A$ es la amplitud pico-pico de las variaciones en la reflectividad. Se observa que dicha señal es una señal cosenoidal, presentando un primer máximo en $v=0$ y periodo $P_{v}=c /(2 h \cos \theta)$ inversamente proporcional al espesor óptico $h$. El argumento de la función coseno $(\phi)$ representa la diferencia de fase ente el haz incidente y el haz reflejado. Es habitual también representar esta señal en función del número de ondas $\tilde{v}=1 / \lambda$ (ó $\tilde{v}=v / c$ ):

$$
R(\tilde{v}, h)=R_{\text {med }}+\frac{A}{2} \cos (4 \pi \tilde{v} h \cos \theta) .
$$

Esta señal alcanza valores extremos (máximos o mínimos) en $v_{m}$ cuando:

$$
\frac{v_{m}}{c /(2 h \cos \theta)}=\frac{m}{2}
$$

ó

$$
v_{m}=m \frac{c}{4 h \cos \theta},\left(\tilde{v}_{m}=\frac{m}{4 h \cos \theta}\right)
$$

Si $m$ es par se está ante un máximo y si $m$ es impar ante un mínimo. La señal interferencial se registra sin capa biológica $\left(h=h_{0}\right)$ y con capa biológica $\left(h=h_{1}\right)$ (ver Fig. 1), observándose un desplazamiento $\Delta v_{m}$ en el extremo (máximo o mínimo) $m$ :

$$
\begin{aligned}
& \frac{v_{m, 0}}{c /\left(2 h_{0} \cos \theta\right)}=\frac{m}{2}, \\
& \frac{v_{m, 1}}{c /\left(2 h_{1} \cos \theta\right)}=\frac{m}{2},
\end{aligned}
$$

$$
\Delta v_{m}=v_{m, 0}-v_{m, 1},
$$

Operando se obtiene:

$$
\begin{aligned}
\Delta v_{m} & =m \frac{c}{4 \cos \theta}\left(\frac{1}{h_{0}}-\frac{1}{h_{1}}\right)= \\
& =m \frac{c}{4 \cos \theta} \frac{\Delta h}{h_{0} h_{1}},
\end{aligned}
$$

donde $\Delta h$ es la diferencia de espesores ópticos $\Delta h=h_{0}-h_{1}$. Con el fin de simplificar se denominará "espesor óptico medio" a la cantidad $\tilde{h}=\sqrt{h_{0} h_{1}}$. Por tanto:

$$
\begin{aligned}
& \Delta v_{m}=m \frac{c}{4 \cos \theta} \frac{\Delta h}{\tilde{h}^{2}}, \\
& \Delta \tilde{v}_{m}=m \frac{1}{4 \cos \theta} \frac{\Delta h}{\tilde{h}^{2}} .
\end{aligned}
$$

La cantidad $C$ de material óptico depositado es, con bastante buena aproximación, una función lineal del incremento de espesor óptico $\Delta h$. Por tanto, podría utilizarse la siguiente expresión donde $\alpha$ es una constante de proporcionalidad (a determinar durante la calibración del sensor frente a materiales de referencia certificados $u$ otros patrones equivalentes con trazabilidad adecuada):

$$
C=\alpha \Delta h .
$$

Pero $\Delta h$ no se mide directamente, lo que se mide es $\Delta v_{m}$; por tanto:

$$
\begin{gathered}
C=\alpha\left(\frac{1}{m} \frac{4 \cos \theta}{c} \tilde{h}^{2}\right) \Delta v_{m}= \\
=c_{\Delta v} \Delta v_{m},
\end{gathered}
$$

donde $c_{\Delta v}$ es el coeficiente de sensibilidad (ver Ref. [3], apartado 5.1.3) de $C$ respecto de $\Delta v_{m}$. La sensibilidad (ver Ref. [7], 4.12) $S$ del sensor sería el inverso de $c_{\Delta v}$. En el caso de trabajar con el número de ondas $\tilde{v}$ se obtendría la siguiente expresión, donde $c_{\Delta \widetilde{v}}$ representaría el coeficiente de sensibilidad de $C$ respecto de $\tilde{v}$ :

$$
\begin{aligned}
C=\alpha \Delta h= & \alpha\left(\frac{1}{m} 4 \cos \theta \tilde{h}^{2}\right) \Delta \tilde{v}_{m}= \\
& =c_{\Delta \widetilde{v}} \Delta \tilde{v}_{m} .
\end{aligned}
$$

\section{Detección del máximo (o mínimo) de la señal}

El objetivo es detectar el mínimo o máximo $x^{*}$ de una señal del tipo $y=y(x)$ de la cual se conocen un conjunto de puntos $\left(x_{i}, y_{i}\right)$. Se posee una 
estimación razonable $x_{0}$ de $x^{*}$ y para detectar el mínimo/máximo se va a proceder a ajustar una parábola a los puntos $\left(x_{i}, y_{i}\right)$ que están situados en el entorno del punto $x_{0}$, más concretamente aquellos puntos para los cuales $\left|x_{i}-x_{0}\right| \leq a$. Se supondrá que se utilizan los puntos $x_{i}=$ $\left(x_{i}-a\right)+[2 a /(n-1)](i-1), \quad i=1,2, \ldots, n$, que se encuentran en el intervalo $\left|x_{i}-x_{0}\right| \leq a$, siendo $n$ el número total de puntos utilizados $\mathrm{y}$ $E_{x}=2 a /(n-1)$ el espaciado entre puntos o resolución. El ajuste de la parábola podría ser descrito del siguiente modo, donde $b_{1}, b_{2}, b_{3}$, son las incógnitas a determinar:

$$
b_{1}+b_{2}\left(\frac{x_{i}-x_{0}}{a}\right)+b_{3}\left(\frac{x_{i}-x_{0}}{a}\right)^{2} \cong y_{i},
$$

para $i=1,2, \ldots, n$, ó

$$
b_{1}+b_{2} w_{i}+b_{3} w_{i}^{2} \cong y_{i}
$$

donde

$$
w_{i}=\frac{x_{i}-x_{0}}{a} .
$$

El sistema de ecuaciones anterior puede ser reescrito en formato matricial del modo siguiente, donde se ha utilizado la expresión $w_{i}=-1+[2 /(n-1)](i-1)$ :

$$
\mathbf{A} \cdot \mathbf{b}=\mathbf{y}
$$

donde:

$$
\begin{aligned}
\mathbf{A} & =\left[\begin{array}{ccc}
1 & w_{1} & w_{1}^{2} \\
1 & w_{2} & w_{2}^{2} \\
& \vdots & \\
1 & w_{n-1} & w_{n-1}^{2} \\
1 & w_{n} & w_{n}^{2}
\end{array}\right]= \\
& =\left[\begin{array}{ccc}
1 & -1 & 1 \\
1 & -1+\frac{2}{n-1} & \left(-1+\frac{2}{n-1}\right)^{2} \\
& \vdots & \\
1 & 1-\frac{2}{n-1} & \left(1-\frac{2}{n-1}\right)^{2} \\
1 & 1 & 1
\end{array}\right],
\end{aligned}
$$

$\mathrm{y}$

$$
\begin{gathered}
\mathbf{b}=\left[\begin{array}{c}
b_{1} \\
b_{2} \\
b_{3}
\end{array}\right], \\
\mathbf{y}=\left[\begin{array}{c}
y_{1} \\
y_{2} \\
\vdots \\
y_{n-1} \\
y_{n}
\end{array}\right] .
\end{gathered}
$$

La solución que proporciona un ajuste por mínimos cuadrados ordinarios [6] al problema anterior es la que se muestra a continuación, donde $\mathbf{U}_{b}$ es la matriz de covarianzas (ver GUMS2 [2], apartado 3.20) del vector $\mathbf{b}$ de incógnitas y $s_{e}$ es el residuo medio cuadrático después del ajuste:

$$
\mathbf{b}=\left(\mathbf{A}^{T} \mathbf{A}\right)^{-1} \mathbf{A}^{T} \mathbf{y}=\left(\mathbf{A}^{T} \mathbf{A}\right)^{-1} S_{e}^{2} .
$$

Cuando el número de puntos es muy elevado, situación relativamente habitual (por ejemplo, la señal completa de la Fig. 1 contiene $1,2 \times 10^{4}$ puntos y en la zona del mínimo cercano a 360 $\mathrm{THz}$ se podrían utilizar del orden de 600 puntos), la matriz $\left(\mathbf{A}^{T} \mathbf{A}\right)^{-1}$ podría aproximarse mediante la siguiente expresión:

$$
\left(\mathbf{A}^{T} \mathbf{A}\right)^{-1} \cong \frac{1}{n}\left[\begin{array}{ccc}
9 / 4 & 0 & -15 / 4 \\
0 & 3 & 0 \\
-15 / 4 & 0 & 45 / 4
\end{array}\right]
$$

y por tanto:

$$
\begin{aligned}
\mathbf{U}_{b}=\left(\mathbf{A}^{T} \mathbf{A}\right)^{-1} & s_{e}^{2} \cong \\
& \cong \frac{1}{n}\left[\begin{array}{ccc}
9 / 4 & 0 & -15 / 4 \\
0 & 3 & 0 \\
-15 / 4 & 0 & 45 / 4
\end{array}\right] s_{e}^{2} .
\end{aligned}
$$

Para hallar el mínimo o máximo $x^{*}$ habría que derivar e igualar a cero la expresión de la parábola ajustada:

$$
y^{\prime}\left(x^{*}\right) \cong \frac{b_{2}}{a}+2 b_{3} \frac{x^{*}-x_{0}}{a^{2}}=0,
$$

de donde se obtiene:

$$
x^{*}=x_{0}-\frac{b_{2}}{2 b_{3}} a=x_{0}-\frac{b_{2}}{2 b_{3}} \frac{(n-1) E_{x}}{2} .
$$

Propagando incertidumbres en la expresión anterior y sabiendo que $u\left(b_{2}, b_{3}\right)=0$ (ver Ec. (21)):

$$
\begin{aligned}
u^{2}\left(x^{*}\right) & =u^{2}\left(x_{0}\right)+ \\
& +\left[\frac{b_{2}}{4 b_{3}}(n-1)\right]^{2} u^{2}\left(E_{x}\right)+ \\
& +\left(\frac{a}{2 b_{3}}\right)^{2} u^{2}\left(b_{2}\right)+\left(\frac{a b_{2}}{2 b_{3}^{2}}\right)^{2} u^{2}\left(b_{3}\right) .
\end{aligned}
$$

Si $x_{0} \cong x^{*}$, puede comprobarse que $y^{\prime}\left(x=x_{0} \cong x^{*}\right) \cong b_{2} / a \cong 0$. Por tanto, desaparecerían las contribuciones asociadas a $E_{x}$ y $b_{3}$. Por otro lado, la componente $u\left(x_{0}\right)$ no está asociada al proceso de determinación del mínimo ó máximo, sino a la trazabilidad de las 
medidas en el eje horizontal. Asimismo, al hallar el desplazamiento $\Delta x^{*}=x_{a}^{*}-x_{b}^{*}$ de estos mínimos o máximos la contribución de $x_{0}$ aparecería tanto en $x_{a}^{*}$ como en $x_{b}^{*}$, desapareciendo. Por ello, la única componente que se retendrá será $u\left(b_{2}\right)$, que además, de acuerdo con la Ec. (21) es $u^{2}\left(b_{2}\right)=3 s_{e}^{2} / n$, esto es:

$$
u\left(x^{*}\right) \cong\left|\frac{a}{2 b_{3}}\right| u\left(b_{2}\right)
$$

$\mathrm{y}$

$$
u\left(\Delta x^{*}\right) \cong \sqrt{2}\left|\frac{a}{2 b_{3}}\right| u\left(b_{2}\right)=\left|\frac{a}{\sqrt{2} b_{3}}\right| \frac{\sqrt{3}}{\sqrt{n}} s_{e} .
$$

El parámetro $b_{3}$ es función de la segunda derivada de la señal en $x_{0} \cong x^{*}, y^{\prime \prime}\left(x^{*} \cong x_{0}\right) \cong$ $2 b_{3} / a^{2}$. Por otro lado, en una señal cosenoidal de periodo $P_{x}$ y amplitud pico pico $A$, dicha segunda derivada toma el valor $y^{\prime \prime}\left(x^{*} \cong x_{0}\right) \cong$ $-2 \pi^{2} A / P_{x}^{2}$. Por consiguiente, $b_{3}=-\pi^{2} A a^{2} / P_{x}^{2}$. Pero $E_{x}=2 a /(n-1)$, de donde $n \approx n-1=$ $2 a / E_{x}$. Por tanto, para este tipo de señales se tendría:

$$
\begin{aligned}
u\left(\Delta x^{*}\right) \cong & \frac{a}{\frac{\pi^{2} A a^{2}}{P_{x}^{2}} \sqrt{2}} \frac{s_{e} \sqrt{3}}{\sqrt{\frac{2 a}{E_{x}}}}= \\
& =\frac{\sqrt{3}}{2 \pi^{2}} \frac{P_{x}^{2}}{a^{3 / 2}} \sqrt{E_{x}} \frac{1}{\mathrm{SNR}},
\end{aligned}
$$

donde SNR (Signal to Noise Ratio en inglés) es la relación señal-ruido $\left(A / s_{e}\right)$. Como se observa, dicha incertidumbre disminuye al crecer la semiamplitud $a$ del intervalo a lo largo del cual se realiza el ajuste. Sin embargo, $a$ no puede crecer indefinidamente, puesto que para valores elevados de a la parábola ya diferiría significativamente del comportamiento de la señal. El error $\varepsilon$ que se comete al aproximar una señal cosenoidal de periodo $P_{x}$ y amplitud picopico $A$ por una parábola alrededor de uno de sus mínimos o máximos es del orden de:

$$
\varepsilon=O\left[\frac{1}{4 !} \frac{A}{2}\left(2 \pi \frac{a}{P_{x}}\right)^{4}\right] .
$$

Con el fin de limitar al máximo el efecto de la posible separación de la parábola frente a la señal cosenoidal se puede fijar el valor máximo de $a$ (y por tanto óptimo) de modo que $\varepsilon$ sea, por ejemplo, del orden de un décimo de $s_{e}$ :

$$
\varepsilon \approx \frac{1}{4 !} \frac{A}{2}\left(2 \pi \frac{a}{P_{x}}\right)^{4}=\frac{s_{e}}{10}
$$

y por tanto:

$$
a_{\text {opt }} \approx \frac{(2 \cdot 4 ! / 10)^{1 / 4}}{2 \pi} P_{x}\left(\frac{S_{e}}{A}\right)^{1 / 4} .
$$

Introduciendo este resultado en la Ec. (26) se obtiene la siguiente expresión:

$$
\begin{aligned}
u\left(\Delta x^{*}\right) \cong & \frac{2 \sqrt{3}}{\sqrt{\pi}(2 \cdot 4 ! / 10)^{3 / 8}} \cdot \\
& \cdot \sqrt{\mathrm{FWHM}_{x} E_{x}} \cdot \mathrm{SNR}^{-\frac{5}{8}}= \\
& =1.09 \sqrt{\mathrm{FWHM}_{x} E_{x}} \cdot \mathrm{SNR}^{-5 / 8},
\end{aligned}
$$

donde $\mathrm{FWHM}_{x}$ (Full Width at Half Maximum) es la anchura de la transición a media altura: $\mathrm{FWHM}_{x}=\gamma P_{x}$. Para señales cosenoidales $\gamma=1 / 2$.

\section{Componente asociada a la detección del máximo (ó mínimo)}

Al propagar incertidumbres en las Ecs. (11), (12), la componente $u_{\Delta v}(C)$ de la incertidumbre de $C$ asociada a la medida de $\Delta v_{m}$ sería $u_{\Delta v}(C)=c_{\Delta v} u\left(\Delta v_{m}\right)$ y por tanto:

$$
\begin{aligned}
u_{\Delta v}(C)=\alpha\left(\frac{1}{m} \cdot \frac{4 \cos \theta}{c} \tilde{h}^{2}\right) & u\left(\Delta v_{m}\right)= \\
& =c_{\Delta v} u\left(\Delta v_{m}\right) .
\end{aligned}
$$

Si para la detección de los extremos $v_{m, 0}, v_{m, 1}$ se utiliza el procedimiento descrito en el apartado 3 , entonces, de acuerdo con la ecuación (30) se obtendría:

$$
u\left(\Delta v_{m}\right)=\beta \sqrt{E_{v} \mathrm{FWHM}_{v}} \cdot \mathrm{SNR}^{-5 / 8},
$$

donde:

- $E_{v}$ es la resolución (distancia en frecuencia entre dos puntos consecutivos),

- $\mathrm{FWHM}_{v}$ es la anchura de la transición,

- SNR es la relación señal-ruido: $\operatorname{SNR}=A / s_{e}$, donde $s_{e}$ es el residuo mínimo cuadrático después de realizar el ajuste por mínimos cuadrados del polinomio. Valores típicos de esta relación señal-ruido pueden estar entre 10 y $10^{4}$. 
- $\beta$ es una constante que depende del tipo de curva que siga la señal. En el caso de cosenoides, $\beta=1,09$.

Introduciendo este resultado en la expresión de la incertidumbre de la cantidad de material biológico:

$$
\begin{aligned}
u_{\Delta v}(C)=\alpha\left(\frac{1}{m}\right. & \left.\cdot \frac{4 \cos \theta}{c} \tilde{h}^{2}\right) . \\
& \cdot \beta \sqrt{E_{v} \mathrm{FWHM}_{v}} \cdot \mathrm{SNR}^{-5 / 8},
\end{aligned}
$$

ó

$$
\begin{aligned}
u_{\Delta v}(C)=\alpha \beta \sqrt{\gamma}\left(\frac{1}{m} \cdot \frac{4 \cos \theta}{c} \tilde{h}^{2}\right) . \\
\cdot \sqrt{E_{v} P_{v}} \cdot \mathrm{SNR}^{-5 / 8} .
\end{aligned}
$$

Anteriormente se ha indicado que $P_{v}=c /(2 h \cos \theta)$. Asimismo, dado que $\Delta h \ll h$ podrá escribirse $h \cong h_{0} \cong h_{1} \cong \tilde{h}=c / 2 P_{v} \cos \theta$. Introduciendo estos cambios en la expresión anterior de $u_{\Delta v}(C)$ y reagrupando términos se obtiene:

$$
\begin{aligned}
& u_{\Delta v}(C)= \\
& \quad=\left(\alpha \beta \sqrt{\gamma} \frac{c}{\cos \theta}\right) \frac{1}{m} P_{v}^{-3 / 2} E_{v}^{1 / 2} \cdot \mathrm{SNR}^{-5 / 8} .
\end{aligned}
$$

Si se trabaja con el número de ondas $\tilde{v}$ en vez de con la frecuencia $v$, la ecuación anterior sería:

$$
\begin{aligned}
& u_{\Delta \widetilde{v}}(C)= \\
& \quad=\left(\alpha \beta \sqrt{\gamma} \frac{c}{\cos \theta}\right) \frac{1}{m} P_{\widetilde{v}}^{-3 / 2} E_{\widetilde{v}}^{1 / 2} \cdot \mathrm{SNR}^{-5 / 8} .
\end{aligned}
$$

donde $P_{\widetilde{v}}=1 /(2 h \cos \theta)$ es el periodo de la señal expresado en número de ondas y $E_{\widetilde{v}}$ es la resolución expresada también en número de ondas.
Por otro lado, el valor de $\tilde{v}=m c /(4 h \cos \theta)$ debe estar cercano al centro $v_{\text {central }}$ del intervalo de trabajo del detector. Si estuviera fuera de dicho intervalo no se podría detectar el mínimo. Por tanto:

$$
\frac{1}{m} \approx \frac{1}{v_{\text {central }}} \cdot \frac{c}{4 h \cos \theta} \text {. }
$$

Introduciendo estos cambios y reagrupando términos de nuevo se obtiene la expresión final para la componente de la incertidumbre asociada a la detección del mínimo/máximo:

$$
\begin{aligned}
& u_{\Delta v}(C)= \\
& =\left(\alpha \beta \sqrt{\gamma} \frac{c}{2 \cos \theta}\right) \frac{1}{v_{\text {central }}} \cdot \frac{E_{v}^{1 / 2}}{P_{v}^{1 / 2}} \cdot \mathrm{SNR}^{-5 / 8} .
\end{aligned}
$$

De nuevo, si se prefiere utilizar el número de ondas $\tilde{v}$ en vez de la frecuencia $v$, la ecuación anterior quedaría en la forma (donde $\left.\tilde{v}_{\text {central }}=v_{\text {central }} / c\right)$ :

$$
\begin{aligned}
& u_{\Delta \widetilde{v}}(C)= \\
& =\left(\alpha \beta \sqrt{\gamma} \frac{c}{2 \cos \theta}\right) \frac{1}{\tilde{v}_{\text {central }}} \cdot \frac{E_{\widetilde{v}}^{1 / 2}}{P_{\widetilde{v}}^{1 / 2}} \cdot \mathrm{SNR}^{-5 / 8} .
\end{aligned}
$$

\begin{tabular}{|c|c|c|c|c|c|c|c|}
\hline \multirow{2}{*}{$\begin{array}{c}\text { Max ó Mín } \\
\text { (THz) }\end{array}$} & \multicolumn{2}{|c|}{ Sin Capa Biológica } & \multicolumn{2}{|c|}{ Con Capa Biológica } & \multirow{2}{*}{$\begin{array}{l}\text { Desplazamiento } \\
\qquad v \pm U_{\Delta v}(\Delta v) \\
\text { (THz) }\end{array}$} & \multirow{2}{*}{$\begin{array}{c}\text { División } \\
\text { de Escala } \\
\text { (THz) }\end{array}$} & \multirow{2}{*}{$\begin{array}{c}\text { Predicción } \\
U_{\Delta v}(\Delta v) \\
(\mathrm{THz})\end{array}$} \\
\hline & $\begin{array}{c}v_{0} \pm U_{\Delta v}\left(v_{0}\right) \\
(\mathrm{THz})\end{array}$ & SNR & $\begin{array}{c}v_{1} \pm U_{\Delta v}\left(v_{1}\right) \\
(\mathrm{THz})\end{array}$ & SNR & & & \\
\hline 310 & $311.95 \pm 0.02$ & $3 \times 10^{3}$ & $309.83 \pm 0.02$ & $5 \times 10^{3}$ & $-2.12 \pm 0.02$ & 0.029 & 0.02 \\
\hline 365 & $366.09 \pm 0.04$ & $4 \times 10^{2}$ & $363.81 \pm 0.04$ & $4 \times 10^{2}$ & $-2.28 \pm 0.05$ & 0.029 & 0.07 \\
\hline 420 & $419.31 \pm 0.06$ & $4 \times 10^{2}$ & $416.95 \pm 0.06$ & $4 \times 10^{2}$ & $-2.36 \pm 0.09$ & 0.029 & 0.06 \\
\hline
\end{tabular}

\section{Resultados y discusión}

Con el fin de mostrar un ejemplo numérico, en la Tabla I se recogen los resultados correspondientes al análisis de las señales representadas en la Fig. 1.

Los resultados correspondientes a las columnas "Sin Capa Biológica", "Con Capa Biológica" y "Desplazamiento" se han obtenido

TABLA I

Resultados correspondientes a las señales de la Fig. 1.

Todas las incertidumbres son incertidumbres expandidas evaluadas para $k=2$ (aprox. 95\%) 
utilizando las técnicas de ajuste por mínimos cuardados descritas en el apartado 2. Las incertidumbres que en ellas aparecen se han estimado de acuerdo con el suplemento 2 de la GUM [2]. En la columna "Predicción" se han incluido las incertidumbres que proporcionaría la ecuación aproximada, Ec. (30). Se comprueba, a partir de los resultados anteriores, que la Ec. (30) predice de forma bastante razonable la componente $u_{\Delta v}(C)$.

Supuestos fijos los siguientes elementos:

- el agente biológico a detectar, es decir, la constante $\alpha$;

- el tipo de curva de la señal (p.e. cosenoidal) y el procedimiento de detección del mínimo (p.e. mediante ajuste por mínimos cuadrados de un polinomio de grado 2), es decir, estarían fijadas las constantes $\beta$ y $\gamma$;

- la relación ruido-señal, SNR;

los únicos parámetros que se podrían variar en esas condiciones serían:

- el periodo de la señal $P_{v}$ a través de la variación del espesor óptico del sensor $h$ (la relación entre ambos es $P_{v}=c /(2 h \cos \theta)$ );

- la resolución $E_{v}$.

En las anteriores condiciones, lo que determina la incertidumbre componente de la incertidumbre $u_{\Delta v}=u_{\Delta v}(C)$ de la cantidad $C$ de agente biológico depositado es la relación $E_{v} / P_{v}$ : la incertidumbre crece con la raíz cuadrada de $E_{v} / P_{v}$. Por tanto, para reducir la contribución de esta componente habría que:

- reducir la resolución $E_{v}$ (siempre y cuando no aparezca correlación entre errores de puntos consecutivos, en cuyo caso ya no serviría de nada seguir reduciendo la resolución);

- incrementar el periodo de la señal $P_{v}$ a través de la reducción del espesor óptico $h$ del sensor. Lo máximo que se podría reducir h de modo que el mínimo obtenido siguiera estando cerca del centro del rango de detección del detector, sería:

$$
\frac{1}{2} P_{v, \max }=v_{\text {central }},
$$

de donde:

$$
h_{\text {min }}=\frac{c}{4 v_{\text {central }} \cos \theta}=\frac{1}{4 \tilde{v}_{\text {central }} \cos \theta},
$$

Para las señales de la Fig. 1, tratando de mantener la posición del mínimo cerca de $v_{\text {central }}=310 \mathrm{THz}$ (que es donde la relación señal-ruido es mejor, del orden de $5 \times 10^{3}$ ) y trabajando $\operatorname{con} \theta=0$, se obtendría $h \approx 240 \mathrm{~nm}$.

\section{Conclusiones}

Utilizando técnicas de ajustes por mínimos cuadrados (las cuales serán objeto de estudio en el documento JCGM 107, [1]), y de propagación de incertidumbres en mensurandos ndimensionales (descritas en el documento JCGM 102:2011, [2]) se ha dado una respuesta metrológica a dos problemas de difícil solución:

- Elección óptima de la semiamplitud a del intervalo a lo largo del cual ajustar una parábola para determinar el mínimo de una señal

- Elección óptima del ancho de la señal $\left(\mathrm{FWHM}_{v}\right)$ cuando ésta puede variarse eligiendo conveniente, por ejemplo, el espesor óptico $h$ del sensor.

El análisis realizado cubre solamente la componente de la incertidumbre asociada a la determinación de los mínimos (o máximos) de la señal. Naturalmente, al tener en cuenta otras componentes, aparecerán otros condicionantes que pueden, quizá, modificar las recomendaciones dadas en el presente artículo. 